\title{
Remark on the muonium to antimuonium conversion in a 3-3-1 model
}

\author{
V. Pleitez \\ Instituto de Física Teórica, Universidade Estadual Paulista, Rua Pamplona, 145, 01405-900- São Paulo, SP, Brazil
}

(Received 19 May 1999; published 7 February 2000)

\begin{abstract}
Here we analyze the relation between the search for muonium to antimuonium conversion and the 3-3-1 model with doubly charged bileptons. We show that the constraint on the mass of the vector bilepton obtained by experimental data can be evaded even in the minimal version of the model since there are other contributions to that conversion. We also discuss the condition for which the experimental data constraint is valid.
\end{abstract}

PACS number(s): 11.30.Fs, 11.30.Hv, 12.60.-i, 36.10.Dr

Recently a new upper limit for the spontaneous transition of muonium $\left(M \equiv \mu^{+} e^{-}\right)$to antimuonium $\left(\bar{M} \equiv \mu^{-} e^{+}\right)$has been obtained [1]. This implies constraints upon the models that induce the $M \rightarrow \bar{M}$ transition. One of them is the 3-3-1 model proposed some years ago [2]. Here we would like to discuss the conditions in which this constraint can be evaded even in the context of the minimal version of the model (minimal in the sense that no new symmetries or fields are introduced).

In that model in the lepton sector the charged physical mass eigenstates (unprimed fields) are related to the weak eigenstates (primed fields) through unitary transformations $\left(E_{L, R}\right)$ as follows:

$$
l_{L}^{\prime}=E_{L} l_{L}, \quad l_{R}^{\prime}=E_{R} l_{R},
$$

where $l_{L}=(e \mu \tau)_{L}^{T}$. It means that the doubly charged vector bilepton, $U_{\mu}^{++}$, interacts with the charged leptons through the current given by

$$
J_{U^{++}}^{\mu}=-\frac{g_{3 l}}{\sqrt{2}} \bar{l}_{L}^{c} \gamma^{\mu} \mathcal{K} l_{L}
$$

where $g_{3 l}$ is the $S U(3)$ coupling constant and $\mathcal{K}$ is the unitary matrix defined as $\mathcal{K}=E_{R}^{T} E_{L}$ in the basis in which the interactions with the $W^{+}$are diagonal $\left(\nu_{L}^{\prime}=E_{L} \nu_{L}\right)$ [3].

In the theoretical calculations of the $M \rightarrow \bar{M}$ transition induced by a doubly charged vector bilepton so far only the case $\mathcal{K}=\mathbf{1}$ has been considered [4]. Although this is a valid simplification, it does not represent the most general case in the minimal 3-3-1 model. In fact, in that model in the quark sector all left-handed mixing matrices survive in different places of the Lagrangian density [5]. In the lepton sector both left- and right-handed mixing matrices survive in the interactions with the doubly charged vector bilepton as in Eq. (2) and also with neutral, doubly and singly charged scalars (see below). Hence, these mixing matrices such as $\mathcal{K}$ in Eq. (2) have the same status as the Kobayashi-Maskawa mixing matrix in the context of the standard model in the sense that they must be determined by experiment. In Ref. [1] it is recognized that their bound is valid only for the flavor diagonal bilepton gauge boson case, i.e., $\mathcal{K}=\mathbf{1}$. If nondiagonal interactions, like in Eq. (2), are assumed, the new upper limit on the conversion probability in the $M \rightarrow \bar{M}$ system implies

$$
M_{U^{++}}>g_{3 l}\left|\mathcal{K}_{\mu \mu}\right|\left|\mathcal{K}_{e e}\right| 2.6 \mathrm{TeV}=850\left|\mathcal{K}_{\mu \mu}\right|\left|\mathcal{K}_{e e}\right| \mathrm{GeV}
$$

If $\left|\mathcal{K}_{\mu \mu}\right|\left|\mathcal{K}_{e e}\right| \approx 0.70$, we get a lower bound of $600 \mathrm{GeV}$ for the doubly charged bilepton which is compatible with the upper bound obtained by theoretical arguments [6].

The following is more important. In addition to the contribution of the vector bileptons there are also the doubly charged and the neutral scalar ones. To consider only the vector bileptons is also a valid approximation since all the lepton-scalar couplings can be small if all vacuum expectation values (VEVs), except the one controlling the $S U(3)$ breaking, are of the order of the electroweak scale and if there is no flavor changing neutral current (FCNC) in the leptonic sector. Both conditions may not be natural in the minimal version of the model, the former because the sextet is introduced only to give mass to the charged leptons so its VEV may be of the order of few $\mathrm{GeV}$, the latter because if we want to avoid FCNC in the lepton sector it is necessary to impose a discrete symmetry which does not belong to the minimal 3-3-1 model. In the model the $S U(3)_{L} \otimes U(1)_{N}$ triplets $\eta=\left(\eta^{0} \eta_{1}^{-} \eta_{2}^{+}\right)^{T} \sim(\mathbf{3}, 0)$ and $\rho=\left(\rho^{+} \rho^{0} \rho^{++}\right)^{T} \sim(\mathbf{3},+1)$ give mass to the quarks. [The third triplet $\chi \sim\left(\chi^{-} \chi^{--} \chi^{0}\right)^{T}$ is out of our concern here.] If the first family transforms in a different way from the other two, the $u$ quark mass is given by the VEV of the $\eta$, here denoted by $\mathrm{v}_{\eta}$; if the third family is that which transforms differently, it is the $t$ quark which gets its mass from $\mathrm{v}_{\eta}$. However, since the mixing matrix of the charge $2 / 3$ quarks is not trivial, the general case interpolates between these two cases. Hence, the vacuum expectation values $\mathrm{v}_{\eta}$ and $\mathrm{v}_{\rho}$ are of the order of the electroweak scale, i.e., $\mathrm{v}_{\eta}^{2}+\mathrm{v}_{\rho}^{2}=(246 \mathrm{GeV})^{2}$. As we said before, the scalar sextet $S \sim(6,0)$,

$$
S=\left(\begin{array}{ccc}
\sigma_{1}^{0} & \frac{h_{2}^{-}}{\sqrt{2}} & \frac{h_{1}^{+}}{\sqrt{2}} \\
\frac{h_{2}^{-}}{\sqrt{2}} & H_{1}^{-} & \frac{\sigma_{2}^{0}}{\sqrt{2}} \\
\frac{h_{1}^{+}}{\sqrt{2}} & \frac{\sigma_{2}^{0}}{\sqrt{2}} & H_{2}^{++}
\end{array}\right),
$$

is necessary in order to give an arbitrary mass to the charged leptons. We will denote $\left\langle\sigma_{2}^{0}\right\rangle=\mathrm{v}_{S}$ the $\mathrm{VEV}$ of the $\sigma_{2}^{0}$, one of 
the neutral components of the sextet. The other one, $\sigma_{1}^{0}$, does not gain a nonzero VEV if the neutrinos must remain massless.

The Yukawa couplings in the lepton sector are

$$
-\mathcal{L}_{l}=\frac{G_{a b}}{\sqrt{2}} \overline{\psi_{a i L}}\left(\psi_{b j L}\right)^{c} S_{i j}+\frac{1}{2} \epsilon_{i j k} G_{a b}^{\prime} \overline{\psi_{a i L}}\left(\psi_{b j L}\right)^{c} \eta_{k}^{*}+\text { H.c. },
$$

where $\psi=\left(\nu l l^{c}\right)^{T}$ and $a, b=e, \mu, \tau ; i, j$ are $S U(3)$ indices, $\eta=\left(\eta^{0} \eta_{1}^{-} \eta_{2}^{+}\right)^{T} ; G_{a b}$ and $G_{a b}^{\prime}$ are symmetric and antisymmetric complex matrices, respectively. (The model can have $C P$ violation in the leptonic sector [3].)

We stress once more that this is the minimal 3-3-1 model because if we want to avoid in Eq. (5) the coupling with the triplet, since only the sextet is necessary for giving to all charged leptons a mass, we have to impose a discrete symmetry. (Only in this case there are no FCNC in the charged lepton sector.) Hence, the mass matrix of the charged leptons has the form

$$
M_{l}=\frac{1}{\sqrt{2}}\left(G_{a b} \vee_{S}+G_{a b}^{\prime} \vee_{\eta}\right)
$$

and it is diagonalized by the bi-unitary transformation $E_{L}^{\dagger} M_{l} E_{R}=\operatorname{diag}\left(m_{e}, m_{\mu}, m_{\tau}\right)$ with $E_{L}$ and $E_{R}$ defined in Eq. (1). However, the bi-unitary transformation does not diagonalize $G$ and $G^{\prime}$ separately. Thus, we have FCNC and there are Yukawa couplings which are not proportional to the lepton masses.

The model has four singly charged and two doubly charged physical scalars, and four $C P$-even and two $C P$-odd neutral scalars. Let us consider the doubly charged and neutral scalar Yukawa interactions. In the sextet given in Eq. (4), $H_{1}^{++}$is a component of a complex triplet under $S U(2)_{L}$ $\times U(1)_{Y}$ with its neutral partner having a vanishing vacuum expectation value (if neutrinos do not get Majorana masses). There are also a doubly charged $\mathrm{H}_{2}^{++}$which is a singlet of $S U(2)_{L}$ and the neutral Higgs boson $\sigma_{2}^{0}$ which is part of a doublet of $S U(2)_{L}$. Hence from Eq. (5) we have the Yukawa interactions (omitting the singly charged scalars)

$$
\begin{aligned}
-\mathcal{L}_{l}= & \bar{l}_{L} \mathcal{K}_{L L}\left(l_{L}\right)^{c} H_{1}^{--}+\left(\bar{l}^{c}\right)_{L} \mathcal{K}_{R R} l_{R} H_{2}^{++} \\
& +\bar{l}_{L} \mathcal{K}_{L R} l_{R} \sigma_{2}^{0}+\bar{l}_{L} \mathcal{K}_{L R}^{\prime} l_{R} \eta^{0}+\text { H.c. }
\end{aligned}
$$

where we have denoted $\mathcal{K}_{L L}=E_{L}^{\dagger} G E_{L}^{*}, \mathcal{K}_{R R}=E_{R}^{T} G E_{R}$ and $\mathcal{K}_{L R}=E_{L}^{\dagger} G E_{R}, \mathcal{K}_{L R}^{\prime}=E_{L}^{\dagger} G^{\prime} E_{R}$. When $G$ and $G^{\prime}$ are real matrices $\mathcal{K}_{L L}$ and $\mathcal{K}_{R R}$ are Hermitian matrices. Notice that $\mathcal{K}_{L R}$ and $\mathcal{K}_{L R}^{\prime}$ are neither unitary nor orthogonal when $G$ and $G^{\prime}$ are real matrices. We see that since the unitary matrices $E_{L, R}$ diagonalize $M_{l}$ in Eq. (6), $\mathcal{K}_{L L}, \bar{K}_{R L}$ and $\mathcal{K}_{R L}$ are not diagonal matrices; thus their matrix elements are arbitrary and only constrained by perturbation theory, by their contributions to the charged lepton masses and by some purely leptonic processes.

As we said before, since there are already two scalar triplets which give the appropriate masses to the $W^{ \pm}$and $Z^{0}$

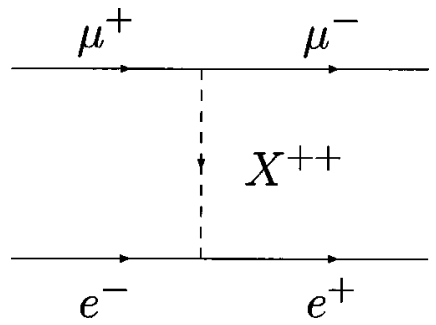

FIG. 1. Contribution to the $M \rightarrow \bar{M}$ conversion. $X^{++}$denotes a vector $U_{\mu}^{++}$or a scalar bilepton $H_{1,2}^{++}$.

vector bosons, it is not necessary that the VEV $\mathrm{v}_{S}$ be of the same order of magnitude as the other vacuum expectation values which are present in the model, $\mathrm{v}_{\eta}$ and $\mathrm{v}_{\rho}$. For instance it is possible that $\mathrm{v}_{S} \approx 10 \mathrm{GeV}$ and $|G| \approx 1$. In this case, the contributions of the doubly charged scalars to the muonium-antimuonium transition can be as important as the contribution of the vector bilepton. There are also new contributions involving FCNC through neutral scalar exchange, which can also give important contributions to the $M \rightarrow \bar{M}$ conversion as has been suggested in Ref. [7]. We see that in the 3-3-1 model all contributions shown in Figs. 1 and 2 do exist. Since there are several contributions to the $M \rightarrow \bar{M}$ conversion, it is still possible to have some cancellations among the scalar and vector bilepton contributions.

In order to appreciate a little more the muoniumantimuonium transition in the 3-3-1 model let us give a brief review of the theoretical results so far known. Many years ago, Feinberg and Weinberg [8] used a $(V-A)^{2}$ Hamiltonian with the four-fermion effective coupling equal to the usual $\beta$-decay coupling constant $C_{V}$, in order to study the $M \rightarrow \bar{M}$ conversion. Let us here denote it by $G_{M \bar{M}}$. The transition amplitude is proportional to $\delta=16 G_{M \bar{M}} / \sqrt{2} \pi a^{3}$ where $a$ is the Bohr radius. More recently, the same transition was studied in the context of models with a doubly charged Higgs boson; in this case the effective Hamiltonian is of the $(V \pm A)^{2}$ form [9-11]. In this case $G_{M \bar{M}}$ is given by the product of two Yukawa couplings $[10,11]$, so in all these cases the sign of the effective coupling $G_{M \bar{M}}$ is undetermined. In models with doubly charged vector bilepton the respective Hamiltonian is of the $(V-A) \times(V+A)$ form [4] with a four-fermion effective coupling given by $G_{M \bar{M}} / \sqrt{2}$ $=-g_{3 l}^{2} / 8 M_{U}^{2}, M_{U}$ being the vector bilepton mass. Hence, in this case always $G_{M \bar{M}}<0$. On the other hand, in $(V \pm A)^{2}$ models the transition amplitude is the same for the singlet

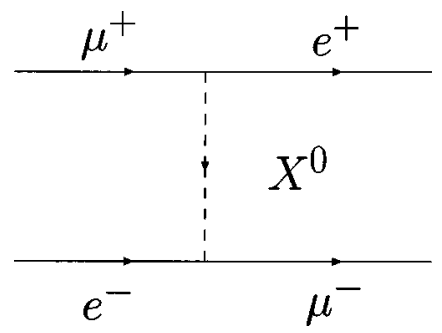

FIG. 2. Contribution to the $M \rightarrow \bar{M}$ conversion. $X^{0}$ denotes a neutral scalar or pseudoscalar. There are also $s$-channel diagrams. 
and triplet muonium given above [8-10] but in $(V-A)$ $\times(V+A)$ models we have $\delta=-8 G_{M \bar{M}} / \sqrt{2} \pi a^{3}$ for the triplet muonium state and $\delta=24 G_{M \bar{M}} / \sqrt{2} \pi a^{3}$ for the singlet state [4]. It has been also shown that pseudoscalars do not induce conversion for triplet muonium, while both pseudoscalars and scalars contribute for singlet muonium. Separate measurements of singlet vs triplet $M \rightarrow \bar{M}$ conversion probabilities can distinguish among neutral scalar, pseudoscalar and doubly charged Higgs induced transitions [7]. Such measurements can also distinguish doubly charged vector bileptons from scalar contributions.

In the 3-3-1 model, besides the doubly charged bosons, there are neutral scalars and pseudoscalars which, as we said before, have flavor changing neutral interactions in the lepton sector. Recall that a scalar (pseudoscalar) mediated effective interaction has opposite (equal) sign with respect to a vector mediated interaction. However, in addition to the $\mathcal{K}$ mixing matrices in Eqs. (2) and (7), there are the mixing matrices among scalars since the scalar fields in Eq. (7) are still weak eigenstates. All these mixing matrices will affect the value and the sign of the effective couplings; hence a cancellation is in fact possible among the contributions to the $M \rightarrow \bar{M}$ transition due to scalars and others due to psedoscalar and vector mediated effective interactions.

The $M \rightarrow \bar{M}$ transition also can be measured in matter. In this case the collisions make the amplitude add incoherently [8]. However, in matter the conversion is strongly suppressed mainly because of the loss of symmetry between $M$ and $\bar{M}$ due to the possibility of $\mu^{-}$transfer in collisions involving $\bar{M}[8,12,13]$. All those data together when available will allow one to constrain models with several sorts of fields inducing the muonium-antimuonium transition.

If all these effects are present or not in the 3-3-1 model, it depends on the value of the parameters. We have argued above that this may be the fact since there are flavor changing neutral interactions in the Higgs-boson-lepton sector and also one of the vacuum expectation values may be of the order of some GeVs.

It is usually considered that the $\mu \rightarrow e \gamma$ decay imposes stronger constraints on a given model than the $M \rightarrow \bar{M}$ transition [11]. However, in the 3-3-1 model the interactions which induce the $\mu \rightarrow e \gamma$ decay involve different mixing matrix elements, and the decay is dominated by the $\tau$ lepton contributions; thus it implies strong constraints on the mixing angles involving this lepton. Another potential trouble for the model is the $\mu \rightarrow e e e$ decay. In order to suppress this decay, the dominant elements of the mixing matrices in Eq. (7) must be the non-diagonal ones.

Summarizing, the bound of Ref. [1] is applied only for a range of parameters in the model and if the sextet is the only Higgs boson which couples to leptons. In this case the neutral current given in Eq. (7) is diagonal and there is no FCNC in the lepton sector and all the sextet-lepton couplings are proportional to $\mathcal{K}_{R L}=\sqrt{2} \hat{M}_{l} / \mathrm{v}_{S}$ where $\hat{M}_{l}$ is the diagonal lepton mass matrix. If $\mathrm{v}_{S}$ is of the order of $100 \mathrm{GeV}$, the main contribution to the $M \rightarrow \bar{M}$ conversion comes from the interaction in Eq. (2) and it constrains the mass of the $U$-vector boson and the mixing angles of the matrix $\mathcal{K}$ as discussed early. However, in general, in the minimal 3-3-1 model the contributions in Figs. 1(a), 1(c) and 1(d) of Ref. [1], here summarized in Figs. 1 and 2, do exist and their experimental data do not, in straightforward way, apply to the model. The $M \rightarrow \bar{M}$ transition deserves indeed more experimental studies.

Experimental difficulties apart, this system in a vacuum could be useful for studying $C P$ and $T$ invariance in the lepton sector: by comparing $M \rightarrow \bar{M}$ with $\bar{M} \rightarrow M$ transitions as has been done recently in the $K^{0} \rightarrow \bar{K}^{0}$ and $\bar{K}^{0} \rightarrow K^{0}$ cases [14].

Finally a remark. The 3-3-1 model predicts that there exists an energy scale, say, $\mu$, at which the model loses its perturbative character. Hence, if experimental data impose a lower bound on the mass of a vector boson of the model which is larger than $\mu$, we can be assured that the model is ruled out. The value of $\mu$ is calculated through the condition $\sin ^{2} \theta_{W}(\mu)=1 / 4$. However, it is not clear at all what the value of $\mu$ is; in fact, it has been argued that the upper limit on the vector bilepton masses is $3.5 \mathrm{TeV}$ [15] instead of the 600 $\mathrm{GeV}$ given in Ref. [6]. Anyway the important thing is that in this model the "hierarchy problem," i.e., the existence of quite different mass scales, is less severe than in the standard model and its extensions since no arbitrary mass scale (say, the Planck scale) can be introduced in the model. This feature remains valid when we introduce supersymmetry in the model [16]. Thus, the breaking of supersymmetry is also naturally at the $\mathrm{TeV}$ scale in this 3-3-1 model.

This work was supported by Fundação de Amparo à Pesquisa do Estado de São Paulo (FAPESP), Conselho Nacional de Ciência e Tecnologia (CNPq) and Programa de Apoio a Núcleos de Excelência (PRONEX).
[1] L. Willmann et al., Phys. Rev. Lett. 82, 49 (1999); B. Ni et al., Phys. Rev. D 48, 1976 (1993).

[2] F. Pisano and V. Pleitez, Phys. Rev. D 46, 410 (1992); P. M. Frampton, Phys. Rev. Lett. 69, 2889 (1992).

[3] J. T. Liu and D. Ng, Phys. Rev. D 50, 548 (1994).

[4] H. Fujii, Y. Mimura, K. Sasaki, and T. Sasaki, Phys. Rev. D 49, 559 (1994); K. Horikawa and K. Sasaki, ibid. 53, 560 (1996).

[5] D. Gómez Dumm, F. Pisano, and V. Pleitez, Mod. Phys. Lett.
A 9, 1609 (1994).

[6] P. Frampton, Int. J. Mod. Phys. A 13, 2345 (1998).

[7] W.-S. Hou and G.-G. Wong, Phys. Rev. D 53, 1537 (1996).

[8] G. Feinberg and S. Weinberg, Phys. Rev. Lett. 6, 381 (1961); Phys. Rev. 123, 1439 (1961).

[9] A. Halprin, Phys. Rev. Lett. 48, 1313 (1982).

[10] M. S. Swartz, Phys. Rev. D 40, 1521 (1989).

[11] D. Chang and W.-Y. Keung, Phys. Rev. Lett. 62, 2583 (1989).

[12] D. L. Morgan, Jr. and V. W. Hughes, Phys. Rev. D 2, 1389 
(1970); Phys. Rev. A 7, 1811 (1973).

[13] However, to have a sensitivity in matter close to that achieved in vacuum at present, muon beams more intensive even than that of the future muon collider will be needed. I am grateful to K. Jungmann for calling my attention to this fact. Anyway, we think it is worth pointing out this possibility.

[14] See http://fnphyx-www.fnal.gov/experiments/ktev/ktev.html.

[15] P. Jain and S. D. Joglekar, Phys. Lett. B 407, 151 (1997).

[16] H. N. Long and P. B. Pal, Mod. Phys. Lett. A 13, 2355 (1998). 